Proceedings of the 42th "Jaszowiec" International School and Conference on the Physics of Semiconductors, Wisła 2013

\title{
The Puzzle of the 0.7 Anomaly in Conductance of Quantum Point Contact Reexamined
}

\author{
T. FIGIELSKI*
}

Institute of Physics, Polish Academy of Sciences, al. Lotników 32/46, 02-668 Warszawa, Poland

\begin{abstract}
A novel approach to the solution of the long-standing problem, called 0.7 anomaly in quantum point contact, is proposed. It is based on fundamental principles of quantum mechanics and can explain all main properties of the anomaly.
\end{abstract}

DOI: 10.12693/APhysPolA.124.841

PACS: 73.23.Ad, 73.61.Ey, 72.25.-b, 03.65.Ge

\section{Introduction}

Apart from usual quantization steps in the conductance of $1 \mathrm{D}$ systems, equal to $G_{0}=2 e^{2} / h$ [1], an additional plateau-like feature appears at a fraction of about 0.7 below the first conductance step in GaAs-based quantum point contacts (QPCs) $[2,3]$. Despite a huge number of research works devoted to this anomalous feature, its origin remains still unclear [4]. Here, an unique explanation of this anomaly is proposed, which relies on the fundamental principles of quantum mechanics: superposition and interference of (spin) states.

\section{Superposition and interference}

Any spin state (spin configuration) of an electron can be represented as a spinor

$$
\left|\psi_{i}\right\rangle=\left(\begin{array}{c}
\cos \left(\theta_{i} / 2\right) \exp \left(-\mathrm{i} \varphi_{i} / 2\right) \\
\sin \left(\theta_{i} / 2\right) \exp \left(\mathrm{i} \varphi_{i} / 2\right)
\end{array}\right),
$$

where $\theta$ and $\varphi$ are the angles of the spherical coordinate system.

Principle of quantum superposition claims that any physical system - such as an electron - exists partially in all its possible states simultaneously, as long as it is not being observed. In the absence of a magnetic field, all spin configurations are equally probable. The superposed spin state of an electron can be then written as $|\psi\rangle=C \sum_{i}\left|\psi_{i}\right\rangle$, where the summation is over all possible spin configurations, and $C$ is a normalization factor. Probability of finding an electron in this state is

$$
P_{\mathrm{S}}=|C|^{2}\langle\psi \mid \psi\rangle=|C|^{2}\left(\sum_{i}\left\langle\psi_{i} \mid \psi_{i}\right\rangle+\sum_{i, j ; i \neq j}\left\langle\psi_{i} \mid \psi_{j}\right\rangle\right) .
$$

The second sum in the bracket results from quantum interference between the different spin states. Because of infinite number of those states, the summations should be replaced by integration over the surface of a unit sphere. Applying that, one finds $P_{\mathrm{s}}=|C|^{2}(1+I)$, where the calculated interference term $I=0.36$.

\footnotetext{
*e-mail: figiel@ifpan.edu.pl
}

While an electron is being observed, the interference term vanishes. Then one has to sum probabilities instead of probability amplitudes, similarly as in case of double-slit interference. The ratio between probability of finding an electron when it is subject to observation, $P_{\mathrm{o}}$, and that when it exists in the superposed state, $P_{\mathrm{s}}$, is $\kappa=P_{\mathrm{o}} / P_{\mathrm{s}}=0.73$.

\section{Destruction of interference in the QPC}

Consider an electron transmitted through a QPC via the lowest 1D energy subband. The electron can exist either in the superposed state (S-regime) or - if it is being observed - in one of the possible spin states (O-regime) that excludes interference. It is known from the double-slit experiment that it is not necessary to perform any real observation of an electron for destructing the interference [5]. It is enough to create conditions allowing such an observation. Here, that condition can appear when no more than one electron at a time travels the constriction region. That condition allows observing a single electron alone. Assuming the constriction represents an 1D channel, the density of electron states is there

$$
\rho=\frac{1}{\pi \hbar}\left(\frac{2 m}{E}\right)^{1 / 2} .
$$

The average number of electrons travelling at the same time through the 1D channel of the length $L$ (in both directions) is

$$
N=\frac{(8 m \mu)^{1 / 2} L}{\pi \hbar},
$$

when the chemical potential (measured from the bottom of the 1D energy subband at the constriction bottleneck) $\mu>k T$.

Assuming $L=50 \mathrm{~nm}$, and $m=0.067 m_{\mathrm{e}}$, one gets $N=1$ for $\mu=0.55 \mathrm{meV}$. As long as the chemical potential remains below this value the system can appear in O-regime, and no interference occurs between different spin states.

\section{Low-temperature limit}

The Heisenberg relation $\Delta x \Delta p \geq \hbar / 2$ determines the uncertainty of electron position $\Delta x$ along the 1D channel, 
where $\Delta p$ is the uncertainty about electron momentum. Requiring that the electron under observation should be fully localized within the constriction, $\Delta x \leq L$, one finds, after some manipulation, a relation that limits the bottom temperature allowing the occurence of O-regime

$$
k T \geq \frac{\mu}{2 \pi} .
$$

The transition from S- to O-regime has to be accompanied by a depression in the conductance of QPC from $G_{0}$ to $\kappa G_{0}$. Thus, just after opening the conducting channel in the constriction its conductance is $\kappa\left(2 e^{2} / h\right)$, and only when the chemical potential exceeds a critical value, the conductance reaches its "normal" magnitude $2 e^{2} / h$. This critical value is gradually reduced as the temperature drops below some level, and eventually a single conductance step at $G_{0}$ is only observed. The calculated value $\kappa=0.73$ is close to the fraction 0.7 characteristic of the observed anomaly.

\section{Summary}

The proposed model has the following advantages over those proposed hitherto: it predicts numerically the fraction of $G_{0}$ characteristic of the anomaly, explains the disappearance of the anomaly at the lowest temperatures, predicts its vanishing with growing length of the constriction, and treats on equal foots both the $n$ - and $p$-type channels.

\section{Acknowledgments}

This work was supported by the National Science Centre (Poland), grant No. 2011/03/B/ST3/02457.

\section{References}

[1] B.J. van Wees, H. van Houten, C.W.J. Beenakker, J.G. Williamson, L.P. Kouwenhoven, D. van der Marel, C.T. Foxon, Phys. Rev. Lett. 60, 848 (1988).

[2] K.J. Thomas, J.T. Nicolls, M.Y. Simmons, M. Pepper, D.R. Mace, D.A. Richtie, Phys. Rev. Lett. 77, 135 (1966).

[3] K.J. Thomas, J.T. Nicolls, N.J. Appleyard, M.Y. Simmons, M. Pepper, D.R. Mace, W.R. Tribe, D.A. Richtie, Phys. Rev. B 58, 4846 (1998).

[4] A.P. Micolich, J. Phys., Condens. Matter 23, 443201 (2011).

[5] S.P. Walborn, M.O. Terra Cunha, S. Pádua, C.H. Monken, Phys. Rev. A 65, 033818 (2002). 